\title{
Effect of probiotic strains upon intestinal immune system under cold stress
}

\author{
Yuri Kozlovsky ${ }^{1}$, Tatiana Khomyakova ${ }^{1, *}$, Aminat Magomedova ${ }^{1}$, Galina Kozlovskaya ${ }^{1}$, \\ and Mariya Zinevich ${ }^{1}$ \\ ${ }^{1}$ FGBNU Institute of Human Morphology, 3, Tsyurupa st., 117418, Moscow, Russia
}

\begin{abstract}
Gut microbiome changes can be considered as a causative factor of stress-associated intestinal diseases. The aim of the investigation was to study the effects of the oral probiotic strains Lactobacillus casei $L B$ 148 and E. coli EB 387 onto gut microbiota, as well as the intestinal immune system of rats Sprague Dawley in a healthy state and under cold stress. There are no strict official recommendations here in the Russian Federation for preclinical studies of new probiotic strains. Here we describe a method used to estimate the safety and effectiveness of the oral use of a probiotic. It is cheap and simple, so we recommend using it at least for the screening search of effective and safe probiotics. The number of Peyer's patches in the small intestine of rats in different compartments was counted and then compared between different groups of rats. After cold stress there was a change in the number of Peyer's patches together with microbiota shifts. Lactobacillus casei $L B 148$ as well as E. coli EB 387 protected the immune system against the stress effects.
\end{abstract}

\section{Introduction}

Cold stress is one of the most significant factors causing the development of an inflammatory process in the body of warm-blooded animals. Studies have shown that acute cold exposure is usually accompanied by the development of a complex of inflammatory and dystrophic changes in internal organs [1]. These changes in rats are usually characterized by the increase in the level of glucocorticoids, catecholamine and $\beta$ endorphin in peripheral blood, adrenal hypertrophy, thymus involution, and ulcerative lesions of the stomach [2]. It was shown that restraint stress reduced the total number of lymphocytes and the number of CD8+ T cells, B cells, and plasma cells in Peyer's patches (PPs); the amount of IgA-producing plasma cells decreased. These effects were due to the effect of glucocorticoids and catecholamines. [3]

Cold stress also affects the functions of the cells of the immune system by modulating phagocytosis, cell proliferation and Th1 / Th2 production of IL-2, IL-4, IFN- $\gamma$, pro- and anti-inflammatory cytokines L $-1 \beta$, TNF- $\alpha$, IL-6, IL-10.Cold stress enhanced levels of IL- 6 . Acute cold stress up-regulated IL-2 and IL-4 levels. IFN-y and IP-10 levels increased significantly [4]. The frequency and duration of exposure, as well as the choice of the model determine the effect of cold stress in an experiment. Chronic stress is associated with

\footnotetext{
*Corresponding author tatkhom@yandex.ru
} 
different disturbances in the structure of the intestinal microbiota and corresponding metabolic shifts in animal experiments, this being also true for humans. A prolonged stress led to a decrease in the level of Lactobacilli and an increase in the level of circulating kynurenine, since metabolic products of lactobacilli can modulate the metabolism of host kynurenine, inhibiting the expression of the metabolizing enzyme IDO1 in the intestine of mice [5]. Cold stress can lead to intestinal inflammation accompanied by changes in the microbiome of mice [6], rats [7] and humans [8]. The changes in the structure of the colon microbiome during acute stress in rats were characterized by a decrease in the number of Lactobacilli, Bifidobacteria and E. coli with normal enzymatic activity [7]. The use of probiotics is prescribed for disbiosis of various genesis, however, it is not known whether the prophylactic use of probiotics before a cold exposure will produce the effect of immunoregulation and reduce the risk of inflammation. The most commonly used probiotic strains are Lactobacilli and Bifidobacteria, while a representative of the normobiota Escherichia coli is seldom used to correct disbiosis, despite its effectiveness. The latter can be confirmed by its inclusion in the Russian Drug Register (OFS.1.7.1.0005.15 Colicontaining probiotics). The successful use of E.coli for the correction the antibiotic-induced disbiosis was previously studied in the work of our laboratory. It was shown that after the oral injection to healthy Vistar rats of E coli EB 387 during one month there weren't any inflammation in the gut of animals, but the use Lactobacillus rhamnosus GG during 30 days a signs of inflammation in the histological slides of the small intestine and colon was found by the light microscopy as well as the shifts of the structure microbiota.

The aim of the experiment was to study comparatively the effects of the oral administration of the probiotic strains Lactobacillus casei LB 148 and E. coli EB 387 on the intestine immune system of Sprague Dawley rats under cold exposure.

\section{Materials and methods}

The study was conducted on male Sprague Dawley rats weighing 100-120 g (4-5 months). The probiotic strains L.casei LB 148 and E. coli EB 387 were used Animals were kept, cared for and taken out of the experiment in accordance with the recommendations of ARRIVE (Animal Research: Reporting of In Vivo Experiments, 2010) and the requirements of the Russian Federation Ministry of Health No. 199n "On Approval of Rules of Good Laboratory Practice" of 2016.

Animals were divided into 6 groups $(\mathrm{N}=5)$ using the pair analogue method. A probiotic preparation at the dose of $1 \times 10^{9} \mathrm{CFU}$ (in $100 \mu \mathrm{l}$ ) was daily administered to rats from days 1 to 21 of the experiment, orally, using an automatic pipette with a metal tube attached to the tip. The animals of groups III and IV received L.casei LB148; the rats of groups V and VI were given E. coli EB 387. The rats in the control group (group I) and group II received oral pyrogen-free physiological saline in the volume of $100 \mu$ for 21 days.

Cold stress was carried out from day 7 to day 21 by placing the rats of groups II, IV and VI in the freezer $\left(\mathrm{t}^{\circ} \mathrm{C}-20-25^{\circ} \mathrm{C}\right)$ for 15 minutes. Thus, the animals of groups IV and VI were subjected to cold stress against the background of the course of the oral probiotics L.casei LB 148 (group IV) and E. coli EB 387 (Group VI), respectively (Table 1)

Table 1. Data on the groups of animals used in the experiment.

\begin{tabular}{|c|c|c|c|c|c|}
\hline Group & $\mathbf{N}$ & Cold stress & $\begin{array}{c}\text { Normal salt } \\
\text { solution }\end{array}$ & $\begin{array}{c}\text { L.casei LB } \\
\mathbf{1 4 8}\end{array}$ & E.coli EB 387 \\
\hline I & 5 & - & + & - & - \\
\hline II & 5 & + & + & - & - \\
\hline III & 5 & - & - & $1 \times 10^{9} \mathrm{KOE}$ & - \\
\hline IV & 5 & + & - & $1 \times 10^{9} \mathrm{KOE}$ & - \\
\hline
\end{tabular}




\begin{tabular}{|c|l|l|l|l|l|}
\hline $\mathrm{V}$ & 5 & - & - & - & $1 \times 10^{9} \mathrm{KOE}$ \\
\hline $\mathrm{VI}$ & 5 & + & - & - & $1 \times 10^{9} \mathrm{KOE}$ \\
\hline
\end{tabular}

The degree of influence of cold stress on the microbiota was estimated in the form of a ratio of Firmicutes to Bacteroides in the fecal boluses of rats by real-time PCR. The number of PP and the present surface area in the small intestine were assessed to estimate the effect of cold stress and probiotic use on the intestinal immune system. The feces were collected on the day before scarification to estimate the qualitative and quantitative composition of the intestinal microbiota. In order to analyze the level of cytokine production using realtime PCR, small and large intestine samples were taken. Statistical data processing was carried out in the program Statistica 6.0 ("Statsoft Inc.", USA). The statistical significance of the differences between groups was determined using the Mann-Whitney U-test. Values $\mathrm{p} \leq 0.05$ were taken as statistically significant. The status of the intestinal immune system was evaluated morphometrically. The small intestine was cut out entirely from the stomach to the cecum, divided into three equal sections - proximal, medial and distal, washed, cut lengthwise, straightened on black paper and photographed.

PP looked like a small oval or round formation visible from the side of the serous membrane of the intestine. The number of Peyer's patches was counted and entered into tables separately for the distal, medial and proximal sections. The area of PP was evaluated using ImagePro Plus 6.RNA from intestinal tissues was isolated in accordance with the instructions for the kit of reagents "ExtractRNA" (Eurogen, Russia).

\section{Results}

According to the results of microbiome analysis, cold stress without probiotic use (group II) induced a decrease in the number of Lactobacilli in the luminal microflora.

The introduction of L.casei LB148 for three weeks (Group III) did not significantly affect the amount of Lactobacilli in the luminal microbiota until the 21 st day of the study, but prevented their decrease in response to cold exposure, which was detected in group II. Daily consumption of E. coli EB387 (group V) for 21 days led to a significant increase in the number of lactobacilli in the absence of cold exposure and had a protective effect on indigenous lactobacilli, compensating for the effect of cold stress on them.

Moreover, the introduction of L.casei LB148 led to a decrease in the ratio of the total share of firmicutes to the share of bacteroids in the luminal microflora. The introduction of E. coli EB 387 (group V) increased the ratio of the Firmicute amount to the Bacteroid one. The results are consistent with the literature on the assessment of the stress effect on the structure of the microbiome in mice [5]. The absence of the effect of probiotic administration to mice not subjected to cold stress is explained by the lack of places for their adhesion in a healthy gut.

When assessing the effect of cold stress on the number of PP in the small intestine over its entire length, the research has shown an increase in their number under the influence of cold stress and a significant decrease in the number $(p<0.05)$ ) with the introduction of probiotics (groups III and V). The use of probiotics protected the development of an immune system reaction. It leads to the absence of differences of the PR number in groups IV and VI (Fig. 1). 


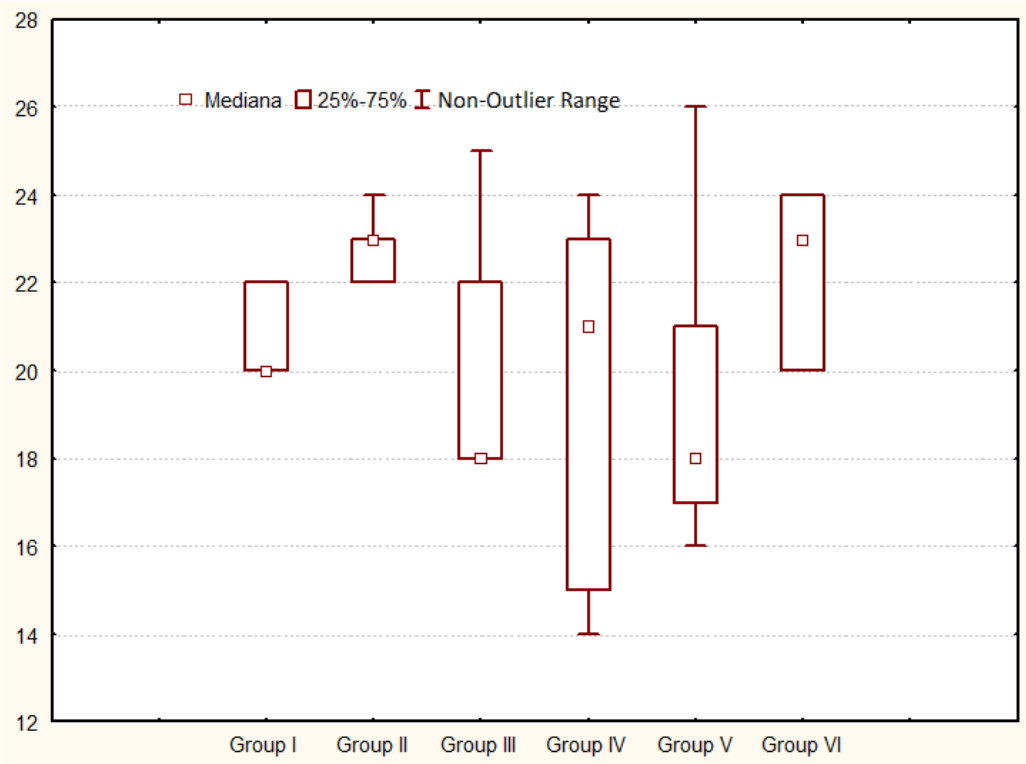

Fig. 1. The common number of the Peyer's patches in the small intestine of rats. The description is in the text.

The analysis of the data on individual compartments has shown that cold stress causes a significant increase in the number of PP, mainly in the proximal small intestine, while in the medial section, this parameter is slightly reduced and in the distal section, it does not differ from the control.

The immune system of the three compartments of the small intestine reacts differently to the administration of Lactobacilli and Escherichia coli: the proximal and medial sections in the group treated with L.casei LB148 did not reveal significant differences from the control (Fig. 2).

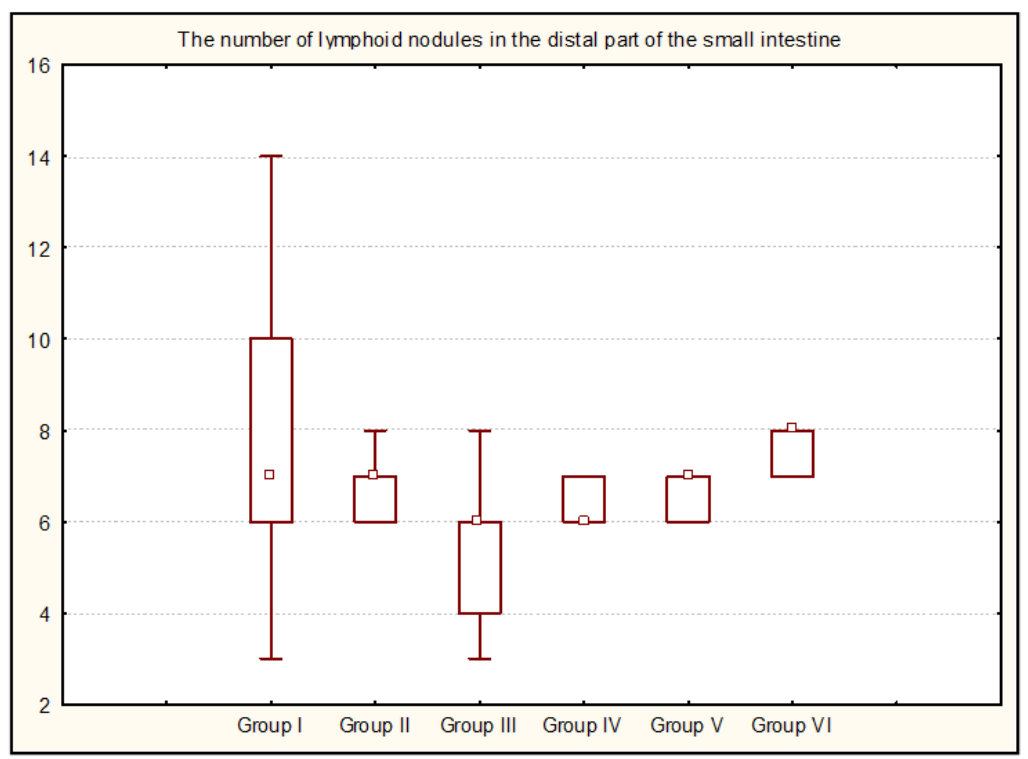

Fig. 2. The number of the Peyer's patches in the distal part of the small intestine. The description is in the text. 
However, the introduction of E.coli EB387 was accompanied by an increase in their number in the proximal section, but did not change in the medial and distal ones. (Fig. 3) The decrease in the number of plaques in the medial section suggests an immunosuppressive effect of lactobacilli, which corresponds to the literature on the antiinflammatory action of the probiotic use. In general, the introduction of lactobacilli does not cause an immune reaction of the small intestine in healthy animals under normal conditions, while E. coli elicits an immune response in the proximal part of the small intestine, which goes out during its further passage through the intestine. The effect of cold stress described above is fully offset by the use of a probiotic based on E. coli EB387 and, to a lesser extent, is prevented by the introduction of lactobacilli.

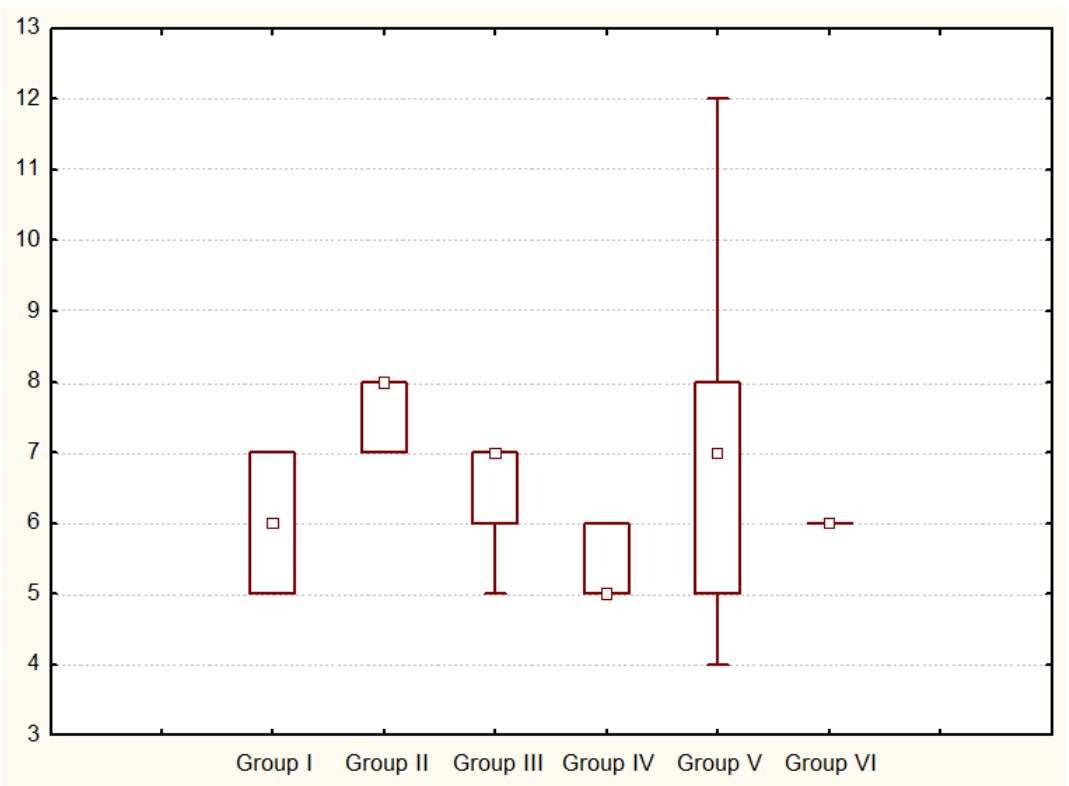

Fig. 3. The number of the Peyer's patches in the proximal part of the small intestine. The description is in the text.

The relative area occupied by PP on the surface of the small intestine (\%) did not significantly differ. However, the absolute values of the total area of PP in the proximal small intestine of rats subjected to cold stress were statistically different from the area of nodules of the rats treated with a probiotic before stress exposure ( $p<0.01)$ (Fig. 4).

This indicator in the distal small intestine of the group II and IV rats receiving lactobacilli was lower than the control.

An analysis of the expression of pro-inflammatory and anti-inflammatory cytokines by group has shown that the L.casei strain had an anti-inflammatory effect in animals exposed to cold stress by increasing the expression of the anti-inflammatory cytokine IL-10 while reducing the expression of IL1- $\alpha$. In the case of oral administration of E. coli strain EB387 under the conditions of cold stress, a significant decrease in IL-10 expression was observed. At the same time, in the absence of cold exposure, its use stimulated the expression of both IL-10 and IL1- $\alpha$. 


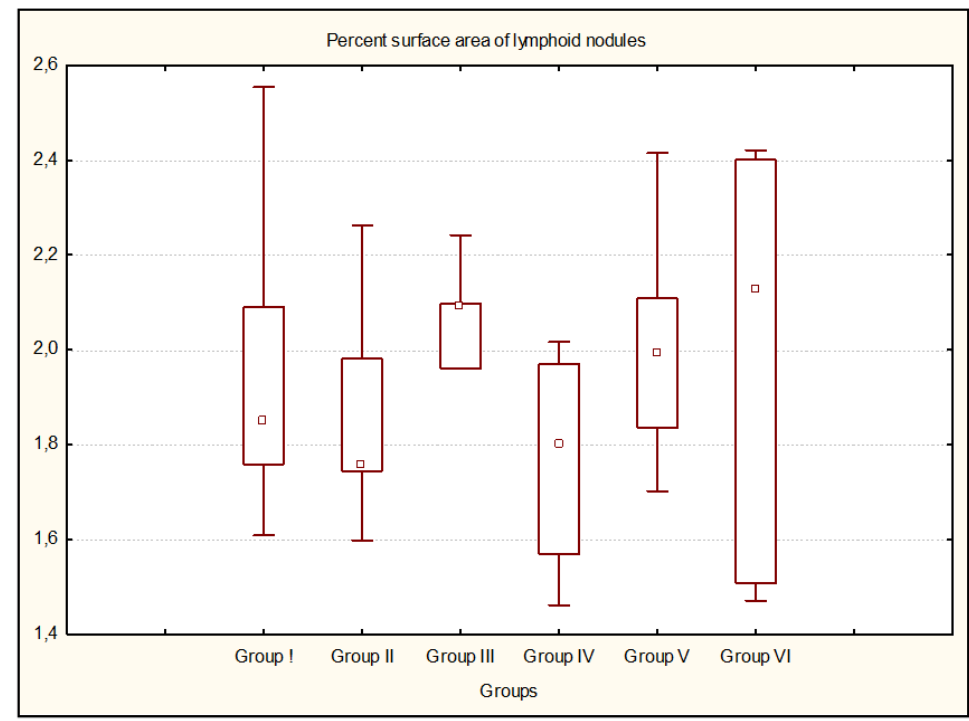

Fig. 4. The relative common area of the Peyer's patches of the small intestine of rats of different groups.

\section{Discussion}

The described above results confirm the regulatory role of bacteria in immune response modulation. The mucosal immune reaction possessa leading roe in the development of immune reaction of the whole body. It was shown the primary role of the mucosal immunity as well as bacterial changes in the development of AIDS, and other socially significant diseases.

First of all we have shown that a severe cold stress lead to the changes of gut microbiota with the specific shifts, characterized by the changes of Firmicutes and Bacteroid. This chages usually normalize during the following month but in the case of the presence of bacterial or viral pathogens it can lead to the development of the disease. So the preventive use of probiotic strains before and just after the cold stress is a very perspective approach to the prevention of the exacerbation of chronic bowel diseases, IBD Crone disease etc.

Stress has a lot of the consequences, commonly attributed to the release of the stress hormone cortisone. Here in our work we have shown that the changes of gut microbiota can be very serious consequences of the stress. Moreover, these changes can be correlated with the use of the oral probiotic use.

It was shown that in mice specific bacteria determine mucosal $\operatorname{IgA}$ production with followed mucosal immune response, they enhance intestinal IgA levels by inducing TGF- $\beta$ in T follicular helper cells of PP Bacteria belonged to Anaeroplasma are sufficient to induce these changes [9]. In rat's PP the indigenous bacteria accelerate the differentiation of microvillous columnar epithelial cells into M-cells [10]. The growth of bacterial colonies between the intervillous spaces associates with the change of histological localization of the lymphocyte lineage in small intestine mucosa of rats [11].

Our date confirm that the lasting stress modulate gut microbiota and the latter influences the host stress response and associated with different complications. That shifts in microbiota structure in human gut were shown at psychological stress, circadian disruption, sleep deprivation and different environmental extremes [12]. Probiotics are highly recommended for the oral use as supplements because of their beneficial effects on health. 
Their characterization based on several properties, including resistance to gastric acidity and bile salt, adherence to mucus and cell lines. They should possess antimicrobial and antagonism activity against pathogenic microbes [13]. There are basic selection criteria established by the World Health Organization (WHO), including hostassociated stress resistance, epithelium adhesion ability, and antimicrobial activity [14].Except of it the results of the last investigations has shown that it is possible to use probiotic strains for a special aims: for wound healing [15], disbiosis protection etc. In those case the probiotic strains should exhibit special properties. The method described above can be used as a cheap method for the additional characterization of new probiotics.

\section{Conclusion}

Thus, the use of the probiotic lactobacilli L.casei LB148 and E. coli EB387 can have a preventive effect against stress-induced disturbances in the structure of the microbiome and subsequent inflammation in the intestine. This allows substantiating their prophylactic and therapeutic effect in human gastrointestinal diseases associated with stress, namely the irritable bowel syndrome, duodenal ulcer and ulcerative colitis. In addition, in preclinical studies the effect of the oral use of a probiotic on the intestinal immune system in the absence of stress and in its presence can serve as indicators of the effectiveness and safety of the biological product. The parameters described in this article can be used as the efficiency indicators.

\section{References}

1. J. Ochoa, B. Malucelli, P. Cruz-Casallas, A. Nasello, F. Luciano, M.I. Carvalho, Neuroimmunomodulation 17, 386-95 (2010) doi:.10.1159/000292063

2. A. Kulikov, L. Arkhipova, D. Kulikov, G. Smirnova, P. Kulikova, Biophysics 58 (2013) doi:10.1134/S0006350913060109

3. M. Beatriz, M. Godínez-Victoria, A. Jarillo-Luna, R. Oros, E. Abarca-Rojano, V. Rivera-Aguilar, J. Pacheco-Yepez, L. Sanchez-Torres, R. Campos-Rodríguez, Neuroimmunomodulation 18, 131-41 (2011) doi: 10.1159/000322625

4. Guo Jing, Li Shi, Fang Hong, Zhang Xu, Wang Jian-Fa, Guo Shuang, Ji Hong, Zang Lin, Guo Li, Zhen Li, Yang Huan, Journal of Animal and Veterinary Advances 11, 1538-1545 (2012) doi:10.3923/javaa.2012.1538.1545

5. C. Kiank, J.-P. Zeden, S. Drude, G. Domanska, G. Fusch, W. Otten, C. Schuett, PLoS ONE 5(7), e11825 (2010) https://doi.org/10.1371/journal.pone.0011825

6. Su-Yeon Joo, Mi-Ju Park, Kyun-Ha Kim, Hee-Jung Choi, Tae-Wook Chung, Yong Jin Kim, Joung Hee Kim, Keuk-Jun Kim, Myungsoo Joo, Ki-Tae Ha, Int. J. Biometeorol 60(8), 1217-25 (2016) doi: 10.1007/s00484-015-1116-5

7. Luo Bin, Shi Hongxia, Zhang Kai, Wei Qiaozhen, Niu Jingping, Wang Junling, Hammond Sally, Liu Sa, Ecotoxicology and Environmental Safety. 168, 9-16 (2019) doi: 10.1016/j.ecoenv.2018.10.064

8. J.P. Karl, L. Margolis, E. Madslien and others, American Journal of Physiology Gastrointestinal and Liver Physiology 312 (2017) doi: ajpgi.00066.2017. 10.1152/ajpgi.00066.2017

9. A. Beller, A. Kruglov, P. Durek and others, Eur. J. Immunol. 17 (2020) doi: 10.1002/eji.201948474

10. H. Yuasa, Y. Mantani, N. Masuda, M. Nishida, M. Arai, T. Yokoyama, H. Tsuruta, J. 
Kawano, N. Hoshi, H. Kitagawa, J. Vet. Med. Sci. 79(11), 1826-1835 (2017) doi: 10.1292/jvms. 17-0470

11. H. Yuasa, Y. Mantani, K. Miyamoto, M. Nishida, M. Arai, H. Tsuruta, T. Yokoyama, N. Hoshi, H. Kitagawa, J. Vet. Med. Sci. 81(4), 555-566 (2019) doi: 10.1292/jvms. 18-0734

12. J.P. Karl, A.M. Hatch, S.M. Arcidiacono, S.C. Pearce, I.G. Pantoja-Feliciano, L.A. Doherty, J.W. Soares, Front. Microbiol. 9, 2013 (2018) doi: 10.3389/fmicb.2018.02013

13. P. Shokryazdan, M. Faseleh Jahromi, J. Boo Liang, Y. Wan Ho, J. Am. Coll. Nutr. 36(8), 666-676 (2017) doi: 10.1080/07315724.2017.1337529

14. G. Vinícius de Melo Pereira, B. Oliveira Coelho, A. Irineudo Magalhães Júnior, V. Thomaz-Soccol, C. Ricardo Soccol, Biotechnol Adv. Dec. 36(8), 2060-2076 (2018) doi: 10.1016/j.biotechadv.2018.09.003

15. N. Han, L. Jia, Y. Su, J. Du, L. Guo, Z. Luo, Y. Liu, Stem. Cell. Res. Ther. Aug. 7.10(1), 243 (2019) doi: 10.1186/s13287-019-1324-8 